\title{
Optimization of electrode geometry and piezoelectric layer thickness of a de- formable mirror
}

\author{
Alexey V. Kruchenko ${ }^{1,2}$, Kateřina Nováková ${ }^{1,2}$, and Pavel Mokrý1,2a \\ ${ }^{1}$ Research Centre for Special Optics and Optoelectronic Systems (TOPTEC), Institute of Plasma Physics, Academy of Sciences \\ of the Czech Republic, Sobotecká 1660, Turnov, CZ-51101, Czech Republic \\ ${ }^{2}$ Institute of Mechatronics and Technical Engineering, Technical University of Liberec, Studentská 2, Liberec, CZ-46117, Czech \\ Republic
}

\begin{abstract}
Deformable mirrors are the most commonly used wavefront correctors in adaptive optics systems. Nowadays, many applications of adaptive optics to astronomical telescopes, high power laser systems, and similar fast response optical devices require large diameter deformable mirrors with a fast response time and high actuator stroke. In order to satisfy such requirements, deformable mirrors based on piezoelectric layer composite structures have become a subject of intense scientific research during last two decades. In this paper, we present an optimization of several geometric parameters of a deformable mirror that consists of a nickel reflective layer deposited on top of a thin lead zirconate titanate (PZT) piezoelectric disk. Honeycomb structure of gold electrodes is deposited on the bottom of the PZT layer. The analysis of the optimal thickness ratio between the PZT and nickel layers is performed to get the maximum actuator stroke using the finite element method. The effect of inter-electrode distance on the actuator stroke and influence function is investigated. Applicability and manufacturing issues are discussed.
\end{abstract}

\section{Introduction}

In the middle of the last century, the resolution of terrestrial astronomical telescopes reached such limits that further improvement of their resolution required a development of methods for the correction of atmospheric distortions. The first concept of so called adaptive optics was envisioned by Babcock [1] in 1953, who proposed to use a deformable mirror to correct the atmospheric seeing. It took more than forty years to achieve a technological level that would allow the construction of adaptive optics.

During the last few decades, several concepts of deformable mirrors were implemented [2]. Examples to be mentioned here are (i) segmented mirrors, (ii) continuous thin plate mirrors, (iii) monolithic mirrors, and (iv) membrane or pellicle mirrors. It is the continuous thin plate type of mirrors, which has become a very popular structure mainly due to their lower technological difficulty. While an array of discrete actuators can always be adjusted to produce zero mean figure error, there is a residual ripple which causes an rms surface error, the magnitude of which can be reduced by closer spacing of actuators. Unfortunately, requirement of dense spacing of the actuators can be satisfied in small and medium size mirrors with many difficulties.

With the onset of real-time wavefront corrections [3], a very convenient type of electromechanical transducers that is used as a actuator in deformable mirrors is the piezo-

\footnotetext{
a e-mail: pavel.mokry@tul.cz
}

electric actuator. The greatest advantage of piezoelectric actuators is their fast response and relatively simple construction. In order to increase the number of degrees of freedom, deformable mirrors based on piezoelectric unimorphs or bimorphs have become a very popular and intensively studied concept [4-8].

Generally, this type of the deformable mirror consists of a layered sandwich composite structure, where the reflective layer is bonded on a piezoelectric layer. The reflective layer is usually made of a conductive metallic material and forms an equipotential surface. On the opposite side of the piezoelectric layer a system of electrodes is deposited using conventional techniques such as lithographic sputtering. By applying a voltage to a particular electrode, the piezoelectric layer is deformed due to the inverse piezoelectric effect in the in-plane directions. This produces bending moments in the reflective layer of the particular segment of a deformable mirror and yield its out-of-plane deformation (see Fig. 1).

The necessity of large voltage-induced deformations is a reason for the use of piezoelectric ceramics as piezoelectric layers. These ceramics are based on a ferroelectric perovskite materials, such as lead zirconate titanate (PZT). Large piezoelectric response of PZT ceramics is achieved due to extrinsic contributions to piezoelectric coefficients due to movements of domain walls in an applied electric field. Since the movements of domain walls are irreversible in high electric fields, the characteristic feature of the actuation is a pronounced hysteresis [8]. This

This is an Open Access article distributed under the terms of the Creative Commons Attribution License 2.0, which permits unrestricted use, distribution, and reproduction in any medium, provided the original work is properly cited. 


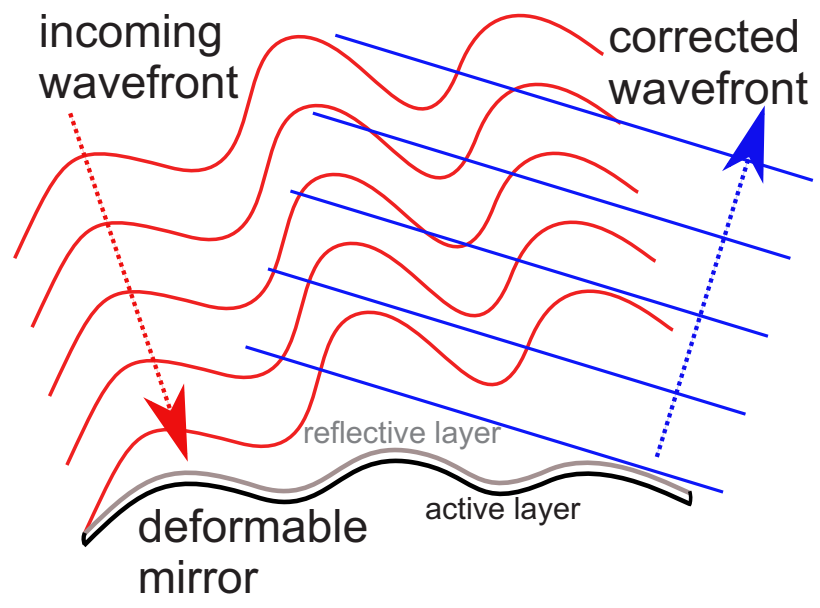

Figure 1. Incoming wavefront, which is distorted by atmospheric turbulence, is reflected from a deformable mirror, which corrects the shape of the wavefront to be planar again. In this work the deformable mirror is designed as a composite structure, where the reflective layer is bonded on the active piezoelectric layer.

strongly hysteretic response represents a source of difficulties in the control of particular deformable mirror segments position. It is clear that reduction or complete elimination of hysteresis would greatly ease the robustness of control algorithms.

There exist two possibilities to reduce hysteresis in the electromechanical response: (i) to use the non-hysteretic piezoelectric material such as electrostrictive or magnetostrictive materials [9], (ii) to reduce the applied electric field to the piezoelectric material while keeping the reasonable piezoelectric response of the whole composite structure.

Aforementioned issues have motivated the work presented below, where we will address the problem of the optimization of the composite structure of the deformable mirror to achieve the maximum out-of-plane deflections at minimum applied voltages to the piezoelectric structure using finite element method (FEM) numerical simulations. In Sec. 2, we will show the geometry of the deformable mirror. Further detail of the FEM model will be presented in Sec. 3. In Sec. 4, we will present the results of our numerical simulation and their discussion. Conclusions will be presented in Sec. 5 .

\section{Geometry of the deformable mirror}

Geometry of the deformable mirror is presented in Fig. 2. The deformable mirror consists of a double-layer sandwich composite structure. In our study, the reflective layer of thickness $h_{\mathrm{Ni}}$ is made of nickel. The reflective nickel layer is bonded on a piezoelectric layer of thickness $h_{\mathrm{PZT}}$ made of a PZT-2. The reflective layer forms an equipotential at the bottom surface of the piezoelectric layer.

On the top surface of the piezoelectric layer, a system of honeycomb golden electrodes is deposited using lithographic sputtering. The distance between the sputtered

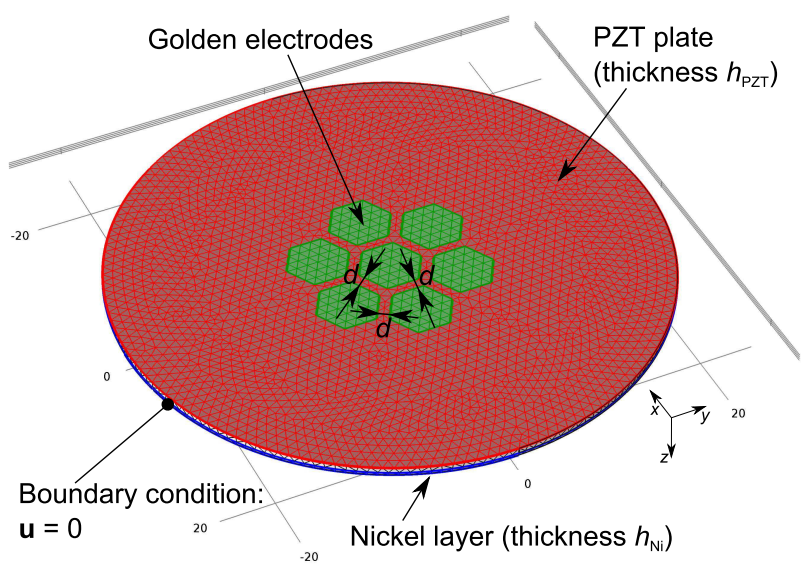

Figure 2. Geometry of the finite element method (FEM) model of the deformable mirror. It consists of the nickel reflective layer of the thickness $h_{\mathrm{Ni}}$ and of the PZT layer of the thickness $h_{\mathrm{PZT}}$. Honeycomb structure of gold electrodes is deposited on the bottom of the PZT layer. Nickel layer itself can be considered as a grounding electrode. The mirror is fixed in its edges which indicates the boundary condition of zero displacement on this edge, i.e. $\mathbf{u}=0$.

electrodes is denoted by a symbol $d$. It is considered that arbitrary external voltage can be applied at each particular electrode. It is considered that the deformable mirror is fixed in a rigid frame along its circumference.

\section{FEM model of the deformable mirror}

The basic equation which rules the analysis is the formula for mechanical stress distribution:

$$
-\nabla \cdot \mathbf{T}=0,
$$

where $\mathbf{T}$ is mechanical stress tensor. Second, we consider the Maxwell equation for the zero flow of electric displacement inside the composite body,

$$
\nabla \cdot \mathbf{D}=0
$$

where $\mathbf{D}$ is electric displacement vector. Electric field $\mathbf{E}$ is given by

$$
\mathbf{E}=-\nabla V,
$$

where $V$ is the electric potential. Third, for the isotropic non-piezoelectric material (i.e. nickel), there are given constitutive equations expressed by the Hooke's law,

$$
\mathbf{S}=\frac{1}{Y} \mathbf{T}-\frac{v}{Y}\left(\mathbf{T}^{T} \mathbf{I}-\mathbf{T}\right)
$$

where $Y$ and $v$ are Young's modulus and Poisson's ratio of an isotropic material, respectively. The symbol I stands for the second-order identity matrix and $\mathbf{S}$ is the elastic strain tensor coupled with the displacement vector $\mathbf{u}$ by the formula:

$$
\mathbf{S}=\frac{1}{2}\left[(\nabla \mathbf{u})^{T}+\nabla \mathbf{u}\right]
$$

In the non-piezoelectric material, the electric displacement is considered as a linear function of the electric field:

$$
\mathbf{D}=\epsilon_{0} \epsilon_{r} \mathbf{E},
$$




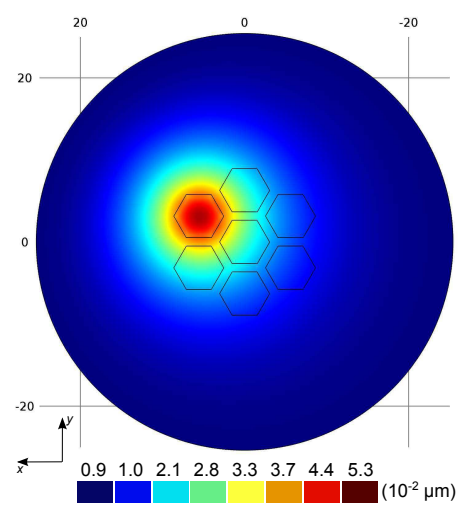

(a)

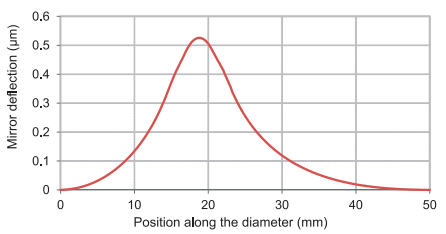

(b)

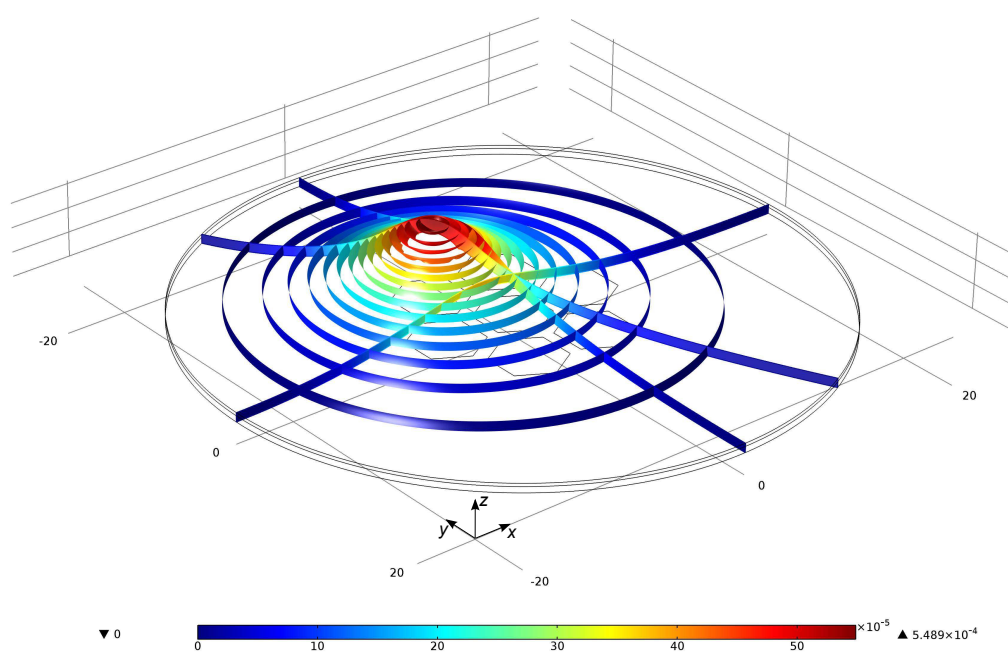

(c)

Figure 3. Graphical presentation of the deformable mirror displacement. One off-centered honeycomb electrode is connected to the electric potential of $200 \mathrm{~V}$, the remaining electrodes are short circuited. The surface boundary between the PZT and nickel layer is taken as a grounding electrode. (a) The 2D surface plot in the plane $(x y)$ of the displacement of the mirror; (b) plot along the line which goes along the diameter of the mirror through the all three honeycomb electrodes; (c) the 3D graphical interpretation of the displacement of the mirror, it is shown using the iso-surfaces and slices. In any case, the color legends mean the mechanical displacement value. The given parameters are $d=1 \mathrm{~mm}, R=3 \mathrm{~mm}, h_{\mathrm{PZT}}=0.5 \mathrm{~mm}$, and the nickel layer thickness $h_{\mathrm{Ni}}=0.18 \mathrm{~mm}$.

where $\epsilon_{r}$ is the relative permittivity of the material. Forth, for the piezoelectric material, the constitutive equations are given by the state equations for the piezoelectric material, written in a following form:

$$
\begin{aligned}
& \mathbf{D}=\left[\epsilon^{T}\right] \mathbf{E}+[d] \mathbf{T} \\
& \mathbf{S}=[d] \mathbf{E}+\left[s^{E}\right] \mathbf{T},
\end{aligned}
$$

where $\left[s^{E}\right]$ are elastic coefficients matrix for constant electric field, $[d]$ is piezoelectric coefficients matrix and $\left[\epsilon^{T}\right]$ is dielectric permittivity matrix at constant mechanical stress.

The mechanical boundary condition, the displacement at the edges of the mirror, is considered as the fixed one, i.e. $\mathbf{u}=0$.

\section{Results and discussion}

Figure 3 shows the example of the FEM numerical simulation of the deformable mirror. In the resented simulation, the off-centered honeycomb electrode is connected to the electric potential of $200 \mathrm{~V}$, the remaining electrodes are short circuited. The following material parameters were considered: $d=1 \mathrm{~mm}, R=3 \mathrm{~mm}, h_{\mathrm{PZT}}=0.5 \mathrm{~mm}$, and the nickel layer thickness $h_{\mathrm{Ni}}=0.18 \mathrm{~mm}$. By applying the FEM model presented in the previous Section, displacements of the deformable mirror were calculated and presented. Figure 3(a) presents the 2D surface plot in the plane $(x y)$ of the displacement of the mirror. Figure 3 (b) presents the plot along the line which goes along the diameter of the mirror through the all three honeycomb electrodes. Figure 3(c) shows the 3D graphical interpretation of the displacement of the mirror, it is shown using the iso-surfaces and slices.

Figure 4 presents results of a similar FEM simulation, however with all honeycomb electrodes connected to the electric potential of $200 \mathrm{~V}$. The remaining numerical parameters are the same as those in Fig. 3. The maximal value of the mirror deflection which could be achieved is $2.02 \mu \mathrm{m}$.

In order to find the optimal ratio of the thicknesses of the nickel and PZT layers, a series of numerical FEM simulations has been performed. At the first step of each simulation, the geometry of the FEM model was modified and the thicknesses of the nickel and PZT layers were set to particular values. In the second step, the displacement of the deformable mirror was calculated. In the third step, the value of the maximum deflection above the activated electrode was determined.

Figure 5 shows the result of the series of simulations, where the maximal values of the mirror deflection is plotted as a function of the nickel layer thickness $h_{\mathrm{Ni}}$ $(0.02-0.6 \mathrm{~mm})$. The parameter of each curve is the PZT layer thickness $h_{\mathrm{PZT}}(0.2-0.8 \mathrm{~mm})$. All the combinations of the different thicknesses of the PZT and nickel layer were used for the finite element method (FEM) model. It can be seen that the thiner layers of both nickel and PZT are the larger displacement of the mirror can be achieved. 


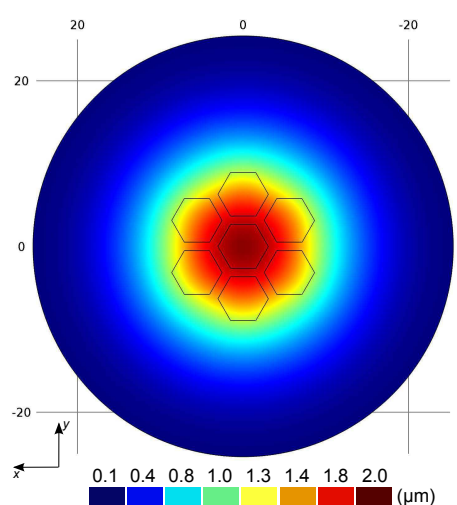

(a)

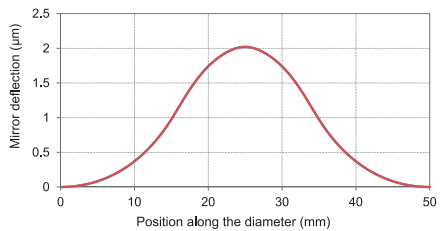

(b)

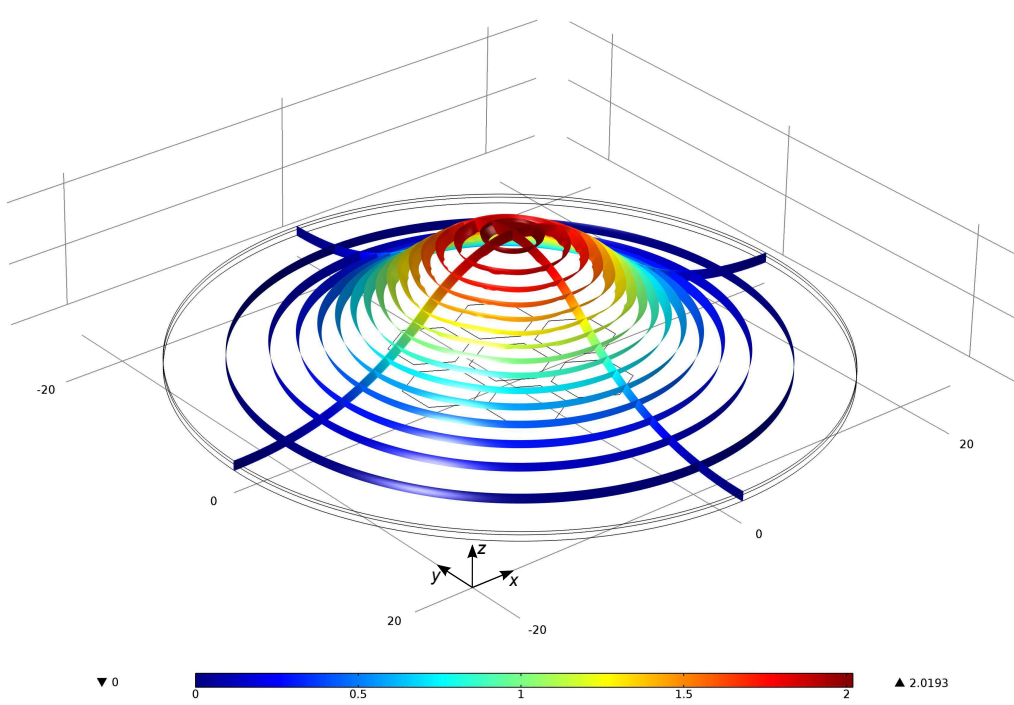

(c)

Figure 4. Graphical presentation of the deformable mirror displacement. All honeycomb electrodes are connected to the electric potential of $200 \mathrm{~V}$. The surface boundary between the PZT and nickel layer is taken as a grounding electrode. (a) The 2D surface plot in the plane $(x y)$ of the displacement of the mirror; (b) plot along the line which goes along the diameter of the mirror through the all three honeycomb electrodes; (c) the 3D graphical interpretation of the displacement of the mirror, it is shown using the iso-surfaces and slices. The remaining numerical parameters are the same as those in Fig. 3. The maximal value of the mirror deflection which could be achieved is $2.02 \mu \mathrm{m}$.

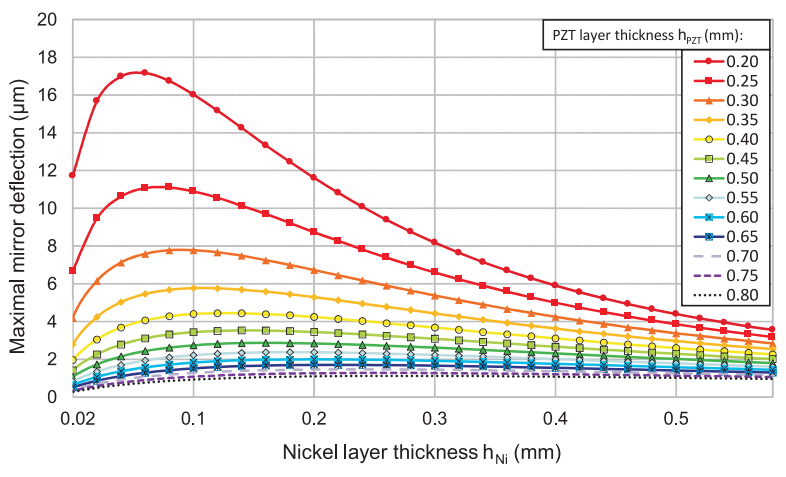

Figure 5. Dependences of the maximal values of the mirror deflection as a function of the nickel layer thickness $h_{\mathrm{Ni}}$ $(0.02-0.6 \mathrm{~mm})$. The parameter of each curve is the PZT layer thickness $h_{\text {PZT }}(0.2-0.8 \mathrm{~mm})$. All the combinations of the different thicknesses of the PZT and nickel layer were used for the finite element method (FEM) model. It can be seen that the thiner layers of both nickel and PZT are the larger displacement of the mirror can be achieved.

Figure 6 presents thickness of the nickel, which results in the maximum deflection of the deformable mirror, versus the thickness of the PZT layer. The dashed line presents the fit of the optimal nickel and PZT thicknesses to the linear dependence obtained by the method of least squares (i.e. the linear regression).

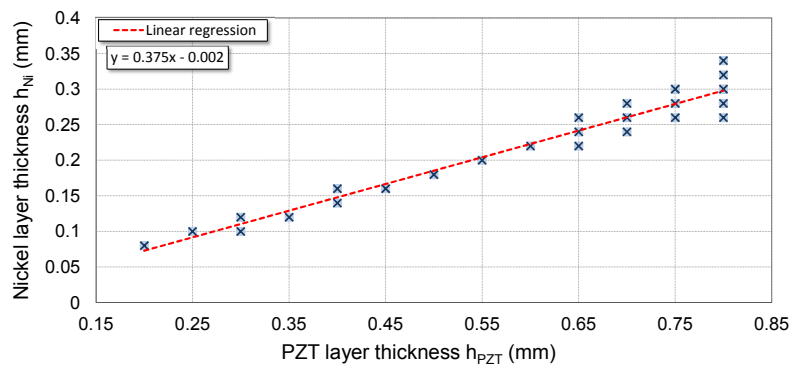

Figure 6. Linear regression of optimal values of the thicknesses of the nickel and PZT layers. The decisive values for the optimization were the maximal values of the mirror displacement which could be seen in Fig. 5. It is shown that for the certain value of the PZT layer thickness could be used certain range of the nickel layer thicknesses, particularly for thicker PZT layers (from $0.65 \mathrm{~mm}$ ).

Figure 7 demonstrates the effect of inter-electrode distance on the deflection of the deformable mirror. The parameter of the curves is the length $R(2.5-3 \mathrm{~mm})$ of the edge of the regular hexagon electrode. All the combinations of the different electrode distances and edge lengths were used for the finite element method (FEM) model. There is a visible maximum in the range of values for the distance between electrodes $(0.5-1 \mathrm{~mm})$. Also, the almost linear dependence on the parameter $R$ could be seen, i.e. 


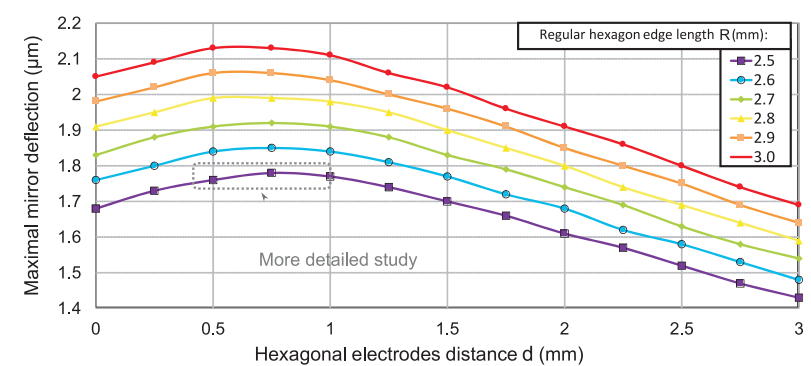

Figure 7. Dependences of the maximal values of the mirror deflection on the honeycomb electrode distance $d(0-3 \mathrm{~mm})$. The parameter of the curves is the length $R(2.5-3 \mathrm{~mm})$ of the edge of the regular hexagon electrode. All the combinations of the different electrode distances and edge lengths were used for the finite element method (FEM) model. There is a visible maximum in the range of values for the distance between electrodes, $(0.5-1 \mathrm{~mm})$. Also, the almost linear dependence on the parameter $R$ could be seen, i.e. the bigger surface of each electrode the bigger deflection of the mirror.

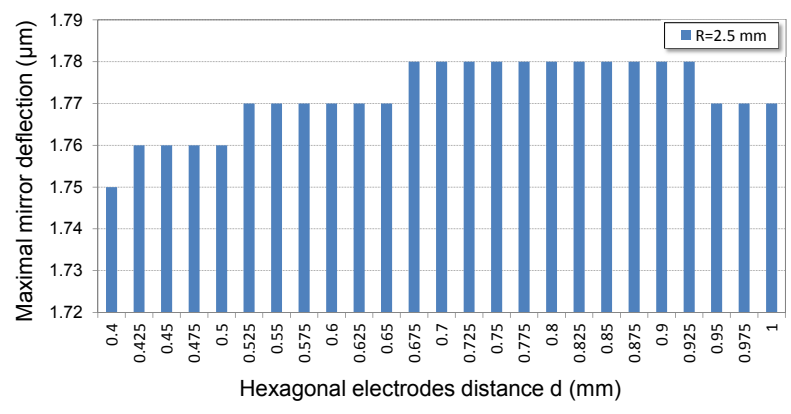

Figure 8. Detailed study of the dependence of the maximal values of the mirror deflection on the honeycomb electrode distance $d$. Very small step of $0.025 \mathrm{~mm}$ was taken into the simulations. The analysis is performed just for the hexagonal electrode edge $R=2.5 \mathrm{~mm}$. The optimal distance between the electrodes is between $d=0.675 \mathrm{~mm}$ and $d=0.925 \mathrm{~mm}$.

the bigger surface of each electrode the bigger deflection of the mirror.

Detailed study of the dependence of the maximal values of the mirror deflection on the honeycomb electrode distance $d$ is presented in Fig. 8. Very small step of $0.025 \mathrm{~mm}$ was taken into the simulations. The analysis is performed just for the hexagonal electrode edge $R=2.5 \mathrm{~mm}$. The optimal distance between the electrodes is between $d=0.675 \mathrm{~mm}$ and $d=0.925 \mathrm{~mm}$.

\section{Conclusions}

FEM model of a deformable mirror, which consists of a double-layer composite structure, was developed. The double-layer composite structure consists of a reflective nickel and a active PZT materials. A series of FEM simulations were performed, in order to find optimal thickness ratio of the reflective and active layers. The effect in interelectrode distances on the maximum deflection of the deformable mirror was analyzed.
The obtained results provide an efficient and simple tool for the design of deformable mirrors with piezoelectric materials. The optimal thickness ratio of the doublelayer composite structure would result in a deformable mirror with minimal hysteresis in the electromechanical response. Such a system allows the use of effortless control algorithms.

\section{Aknowledgments}

This work was supported by the European Regional Development Fund and the Ministry of Education, Youth and Sports of the Czech Republic in the Project No. CZ.1.05/2.1.00/03.0079: Research Center for Special Optics and Optoelectronic Systems (TOPTEC), by the Student Grant SGS 2012/7821 Interactive Mechatronics Systems Using the Cybernetics Principles, and by the Project TA01010878 of Technology Agency of the Czech Republic.

\section{References}

[1] H.W. Babcock, Publ. Astron. Soc. Pac. 65, 229 (1953)

[2] J. Hardy, Proceedings of the IEEE 66, 651 (1978)

[3] F. Merkle, Real-time Wave-front Sensing And Adaptive Optics, in Diffraction-limited Imaging With Very Large Telescopes, edited by Aalloin, Dm and Mariotti, Jm (Nato, Div Sci Affairs; Minist Affaires Etrangeres France; Natl Sci Fdn; Cnrs; European Space Agcy, 1989), Vol. 274 of Nato Advanced Science Institutes Series, Series C, Mathematical And Physical Sciences, pp. 237-248, ISBN 0-7923-0192-7, Nato Advanced Study Inst On Diffraction-limited Imaging With Very Large Telescopes, Cargese, France, Sep 13-23, 1988

[4] H. Zhao, P. Fekete, J. OByrne, Prototype bimorph mirror for the AAT adaptive optics system, in Adaptive Optics And Applications, edited by Tyson, RK and Fugate, RQ (Soc Photo Opt Instrumentat Engineers, 1997), Vol. 3126 of Proceedings Of The Society Of Photo-optical Instrumentation Engineers (SPIE), pp. 384-390, ISBN 0-8194-2548-6, Conference on Adaptive Optics and Applications, SAN DIEGO, CA, JUL 30-AUG 01, 1997

[5] M. Huonker, G. Waibel, A. Giesen, H. Hugel, Fast and compact adaptive mirror, in Lasers in material processing, edited by Beckmann, LHJ (European Opt Soc; Soc Photo Opt Instrumentat Engineers; Commiss European Communities, Directorate Gen Sci Res \& Dev, 1997), Vol. 3097 of Proceedings of the society of photo-optical instrumentation engineers (SPIE), pp. 310-319, ISBN 0-8194-2517-6, Conference on Lasers in Material Processing, MUNICH, GERMANY, JUN 16-20, 1997

[6] R. Signorato, O. Hignette, J. Goulon, Journal of Synchrotron Radiation 5, 797 (1998)

[7] E. Yang, K. Shcheglov, A piezoelectric unimorph deformable mirror concept by wafer transfer for ultra large space telescopes, in Adaptive Optical System Technologies Ii, Pts 1 And 2, edited by Wizinowich, 
PL and Bonaccini, D (SPIE, 2003), Vol. 4839 of Proceedings Of The Society Of Photo-optical Instrumentation Engineers (SPIE), pp. 703-710, ISBN 0-81944618-1, ISSN 0277-786X, Conference on Adaptive Optical System Technologies II, WAIKOLOA, HI, AUG 22-26, 2002
[8] I. Kanno, T. Kunisawa, T. Suzuki, H. Kotera, Selected Topics in Quantum Electronics, IEEE Journal of 13, 155 (2007)

[9] M. Guo, S. Dong, B. Ren, H. Luo, Ultrasonics, Ferroelectrics and Frequency Control, IEEE Transactions on 58, 2737 (2011) 